\title{
Perinatal morbidity and mortality associated with chlamydial infection: a meta-analysis study
}

\begin{abstract}
Objective: To evaluate the effect of Chlamydia trachomatis infection during pregnancy on perinatal morbidity and mortality. Methods: Systematic review and meta-analysis in an electronic database and manual, combining high sensitivity specific descriptors seeking to answer the research objective. The articles considered to be of high methodological quality (score above 6 on the Newcastle-Ottawa Scale) were assessed by meta-analysis. Results: Summary estimates of 12 studies were calculated by means of Mantel-Haenszel test with 95\% confidence interval. It was observed that Chlamydia infection during pregnancy increased risk of preterm labor (relative risk $(\mathrm{RR})=1.35[1.11,1.63]$ ), low birth weight $(\mathrm{RR}=1.52[1.24,1.87])$ and perinatal mortality $(\mathrm{RR}=1.84[1.15,2.94])$. No evidence of increased risk was associated with Chlamydia infection in regard to premature rupture of membranes $(\mathrm{RR}=1.13[0.95,1.34])$, abortion and postpartum endometritis $(\mathrm{RR}=1.20[0.65,2.20]$ and $0.89[0.49,1.61]$ respectively). Conclusion: The diagnosis and treatment of Chlamydia cervicitis during pregnancy can reduce perinatal morbidity and mortality associated with this infection. However, clinical trials are needed to confirm these findings.
\end{abstract}

Keywords: Chlamydia trachomatis; perinatal mortality; morbidity; meta-analysis.

\section{INTRODUCTION}

Chlamydia trachomatis (CT) is responsible for the most prevalent sexually transmitted bacterial infection worldwide. ${ }^{1-4}$ The World Health Organization (WHO) estimates that 92 million new cases occur annually, four million in the United States and 10 million in Europe. ${ }^{1,3-5}$ In Brazil an estimate from the Ministry of Health states approximately two million new cases only in $2001 .^{6}$

Besides high prevalence, another concern is the high annual cost resulting from the sequels of this infection, estimated at four billion dollars, thus achieving second place among the most expensive sexually transmitted diseases (STDs). ${ }^{1,7} \mathrm{CT}$ is usually asymptomatic and persistent, as well as widely distributed throughout the population; especially among the young. ${ }^{8,9}$

On the other hand, in Brazil CT detection is not a routine procedure: it is only recommended when patients show symptoms. This lack of diagnosis contributes considerably to the high prevalence of untreated CT leading to seriously damaging consequences. ${ }^{10}$
In women CT initially infects the cervix and urethra, eventually causing abnormal vaginal discharge and dysuria. When not treated the infection can reach the fallopian tubes, causing pelvic inflammatory disease and adhesions. ${ }^{11-13}$ Abdominal pain, back pain, nausea, fever, dyspareunia, or menorrhagia affect up to $40 \%$ of CT include chronic pelvic pain, infertility and increased incidence of ectopic pregnancy. ${ }^{14-16}$

In pregnant women, $\mathrm{CT}$ is even more critical as it may affect normal intra- and extrauterine development. ${ }^{17}$ The pregnancy itself seems to increase the risk of CT colonization and alter immune response. It may also influence the clinical manifestations of disease in pregnant women. ${ }^{18,19}$ In the United States, $2 \%$ to $13.7 \%$ of pregnant women are carriers of CT. ${ }^{18-22}$ In Brazil some studies have shown prevalence rates of between $2.7 \%$ and $10 \% .^{2,10,23}$ In this group, CT has been associated with an increased risk of preterm delivery, spontaneous abortion, ${ }^{24}$ low birth weight, premature rupture of membranes (PROM) and perinatal mortality. ${ }^{25,26}$ Moreover, children of mothers with CT untreated women. The sequelae resulting from
Authors

Maria José Penna Maisonnette de Attayde Silva ${ }^{1}$

Gilzandra Lira Dantas Florêncio $^{2}$

José Roberto Erbolato Gabiatti ${ }^{3}$ Rose Luce do Amaral ${ }^{4}$ José Eleutério Júnior ${ }^{5}$ Ana Katherine da Silveira Gonçalves $^{6}$

${ }^{1}$ MSc Student, Postgraduate Program in Health Sciences, Universidade Federal do Rio Grande do Norte (UFRN);

Professor, Universidade Potiguar (UnP), RN, Brazil

${ }^{2} \mathrm{MSc}$ Student, Postgraduate Program in Health Sciences, UFRN, RN, Brazil

${ }^{3} \mathrm{PhD}$; Associate Professor and Chief, Tocoginecology Department, Faculdade de Ciências Médicas, Universidade Estadual de Campinas (FCM-UNICAMP), SP, Brazil ${ }^{4} \mathrm{PhD}$ in Tocoginecology, UNICAMP; Physician, UNICAMP, SP, Brazil ${ }^{5} \mathrm{PhD}$ in Ginecology, UNICAMP Associate Professor, Universidade Federal do Ceará (UFC), CE, Brazil

${ }^{6} \mathrm{PhD}$, UNICAMP; Associate Professor, UFRN, RN, Brazil

Submitted on: 03/23/2011 Approved on: 04/13/2011

Correspondence to: Ana Katherine da Silveira Gonçalves

Rua Major Laurentino de Morais, 1218/1301 59020-390 - Natal, SP, Brazil Phone: +55 8436154160 katherin@fcm.unicamp.br

Financial Support: This study was supported by CNPQ Edital MCT/CNPq n 70/2008 Mestrado e Doutorado (\# 554784/2009-2).

We declare no conflict of interest.

(C)2011 Elsevier Editora Ltda. All rights reserved. 
may be infected during passage through the birth canal, developing conjunctivitis and pneumonia. ${ }^{27}$

That being said, studies conducted in pregnant women infected with CT show conflicting results regarding the risk of these events. Knowledge concerning the impact of infection in this group can improve counseling and treatment of infected pregnant women as a public health measure.

The objective of this meta-analysis was to evaluate perinatal morbidity associated with CT infection, determining the risk of preterm labor (PTL), PROM, low birth weight, perinatal mortality, endometritis and abortion.

\section{MATERIAL AND METHODS}

Studies were selected through systematic review to determine the risk of perinatal morbidity and mortality associated with CT infection, and classified according to the outcomes: PTL (labor occurring before 37 weeks gestation), PROM, low birth weight (full term newborn weighing less than 2500 grams), perinatal mortality (mortality occurring between 20 weeks of gestation until 28 days after birth), endometritis (infection of the endometrium or decidua) and abortion (expulsion or fetal death before 20 weeks gestation). Searches were conducted on electronic databases such as PubMed, Embase, Lilacs, Cochrane, and Google Scholar. Manual searches were also conducted through gynecology journals and the bibliographies of reviewed articles.

The first part of the study involved examining major electronic databases. Results were acquired searching by keyword and specific descriptors determined by the database criteria, and combined to increase the sensitivity of the search for articles on the assessment of risk associated to CT infection in pregnancy (Box 1).

Articles were selected by means of eligibility criteria. To be included, a cohort study or case control study needed to be carried out on pregnant women diagnosed with CT during pregnancy who underwent medical follow-up

2695 articles identified in electronic databases and manual search

2550 articles excluded after assessment of titles and abstracts

145 articles with full text assessment

77 excluded articles (did not present adequate study design - cohort or case control)

68 articles with full text assessment

56 excluded articles (did not meet the selection criteria)

13 articles

Loss $=1$

12 articles

Box 1: Article selection process from the search to the quality assessment. before and after childbirth. Publications from January 1980 to January 2010 were considered in English, Portuguese, Spanish or French. Articles were only included following the evaluation and agreement of two reviewers.

The Newcastle-Ottawascale was used to assess the methodological quality of studies. This scale varies between zero and nine points: studies with a score equal to or higher than six were considered methodologically sound.

Data collection of the primary studies was made using the appropriate instrument (the Newcastle scale) in order to obtain a summary measure flow chart. After calculating relative risk using the Mantel-Haenszel with a confidence interval of $95 \%$, tabulation and interpretation of results ensued.

\section{RESULTS}

After a manual search of the databases, 2695 articles were collected. Of these, 2550 articles were excluded when the titles and abstracts were not found to meet eligibility criteria (Box 1).

Among the 145 remaining items, the 12 selected had a score equal to or greater than 6 on the Newcastle-Ottawa scale (Table 1). The selected articles are risk assessment studies, carried out in populations of pregnant women who sought care in prenatal clinics at least until the end of the pregnancy. The diagnostic methods used included culture, molecular biology tests and/or IgM antibodies (Table 1).

CT prevalence ranged from $2.4 \%$ to $35.9 \%$, depending on the characteristics of the population studied and the diagnostic methods used (Table 1). It was observed that CT infection during pregnancy increased the risk of PTL 1.51 $[1.27,1.80]$. The test for heterogeneity was found to be significant when all items were considered in the evaluation. The analysis of funnel plot items from Gravett et al., ${ }^{28}$ Jain et al. ${ }^{29}$ and Martin et al. ${ }^{30}$ were removed for statistical reevaluation, and as a result the relative risk (RR) decreased to $1: 35[1.11,1.63]$ while heterogeneity lost its significance $(\mathrm{p}=0.07)$. (Table 2). The RR of CT infection and PROM was 1.13 [0.95 1:34] and the studies were found to be homogeneous for meta-analysis (heterogeneity $\mathrm{p}=0.10$ ). Similar resultswerefound withlowbirth weight, $\mathrm{RR}=1.52[1.24,1.87]$, among the studies considered homogeneous for evaluation $(\mathrm{p}=0.1)$ (Table 2). Perinatal mortality was found to have a RR of 2.44 [1.63, 3.68], and items were shown to be heterogeneous $(\mathrm{p}=0.02)$. Following the evaluation of the funnel plot, the studies by Jain et al. ${ }^{29}$ and Martin et al. ${ }^{30}$ were removed, since their RR was $1.84[1.15,2.94] \mathrm{p}=1.0$. (Figure 1).

Regarding endometritis and abortion, no significant correlation with $\mathrm{CT}$ in pregnancy was found, $\mathrm{RR}=1.20$ $[0.65,2.20]$ and $R R=0.89[0.49,1.61]$ respectively. Few studies of good quality have addressed such outcomes (Box 1). 
Table 1. Characteristics of risk assessment studies included in meta-analysis

\begin{tabular}{|c|c|c|c|c|c|c|c|c|c|}
\hline Autor & Design & Local & Exclusion criteria & n & Loss & $\begin{array}{c}\text { Time } \\
\text { (months) }\end{array}$ & $\begin{array}{l}\text { Diagnostic } \\
\text { method }\end{array}$ & $\begin{array}{l}\text { P (Chlamydia } \\
\text { trachomatis) } \\
\text { score }\end{array}$ & $\begin{array}{c}\text { Newcastle- } \\
\text { Ottawa }\end{array}$ \\
\hline $\begin{array}{l}\text { Andrews } \\
\text { et al. } .^{20}\end{array}$ & $\begin{array}{l}\text { Cohort } \\
\text { nested } \\
\text { trial }\end{array}$ & EUA & $\begin{array}{c}\text { Pregnants } \\
\text { (16 to } 23 \text { ) } \\
\text { weeks }\end{array}$ & 2570 & 214 & 12 & LCR & $9.9 \%(238: 2398)$ & 8 \\
\hline $\begin{array}{l}\text { Berman } \\
\text { et al. }{ }^{31}\end{array}$ & $\begin{array}{c}\text { Retrospective } \\
\text { cohort }\end{array}$ & India & $\begin{array}{l}\text { Pregnants } \\
<24 \text { weeks }\end{array}$ & 1203 & 104 & 36 & Culture & $22 \%(251 / 1152)$ & 8 \\
\hline $\begin{array}{l}\text { Blas } \\
\text { et al. }{ }^{13}\end{array}$ & $\begin{array}{l}\text { Retrospective } \\
\text { cohort }\end{array}$ & EUA & Pregnants & 4255 & $425^{*}$ & 12 & PCR & - & 7 \\
\hline $\begin{array}{l}\text { Gravett } \\
\text { et al. }{ }^{28}\end{array}$ & $\begin{array}{c}\text { Prospective } \\
\text { cohort }\end{array}$ & EUA & $\begin{array}{c}\text { Pregnants } \\
\text { 2-3 trimester }\end{array}$ & 582 & 28 & 5 & Culture & $9 \%(47 / 534)$ & 6 \\
\hline $\begin{array}{l}\text { Harrison } \\
\text { et al. }\end{array}$ & $\begin{array}{c}\text { Prospective } \\
\text { cohort }\end{array}$ & EUA & $<32$ weeks & 1365 & 180 & 18 & Culture & $8 \%$ ( $94 / 1185)$ & 7 \\
\hline $\begin{array}{l}\text { Jain } \\
\text { et al. }{ }^{29}\end{array}$ & Case-control & India & $\begin{array}{c}\text { Pregnants } \\
3^{\text {rd }} \text { trimeste } \\
\text { to labor }\end{array}$ & 78 & 18 & 3 & $\operatorname{Ig} M$ & $35.9 \%(28 / 78)$ & 6 \\
\hline $\begin{array}{l}\text { Kirk } \\
\text { et al. }{ }^{17}\end{array}$ & $\begin{array}{c}\text { Prospective } \\
\text { cohort }\end{array}$ & London & $\begin{array}{l}\text { Pregnants } \\
<14 \text { weeks }\end{array}$ & 1101 & 295 & 4 & $\begin{array}{l}\text { Vaginal } \\
\text { PCR }\end{array}$ & $2.2 \%(18 / 806)$ & 6 \\
\hline $\begin{array}{l}\text { Kovacs } \\
\text { et al. }{ }^{33}\end{array}$ & $\begin{array}{c}\text { Prospective } \\
\text { cohort }\end{array}$ & Hungary & Pregnants & 6161 & & 18 & $\begin{array}{l}\text { Gen } \\
\text { probe }\end{array}$ & $5.75 \%(362$ /6161) & 7 \\
\hline $\begin{array}{l}\text { Martin } \\
\text { et al. }{ }^{30}\end{array}$ & $\begin{array}{c}\text { Prospective } \\
\text { cohort }\end{array}$ & EUA & $\begin{array}{l}\text { Pregnants } \\
<19 \text { weeks }\end{array}$ & 304 & 36 & 12 & Culture & $6.7 \%(18 / 268)$ & 7 \\
\hline $\begin{array}{l}\text { Oakeshott } \\
\text { et al. }{ }^{34}\end{array}$ & Prospective & $\begin{array}{c}\text { England } \\
\text { cohort }\end{array}$ & $\begin{array}{l}\text { Pregnants } \\
<14 \text { weeks }\end{array}$ & 1216 & 15 & 24 & PCR & $2.4 \%(19 / 1214)$ & 7 \\
\hline $\begin{array}{l}\text { Silveira } \\
\text { et al. }{ }^{35}\end{array}$ & $\begin{array}{l}\text { Retrospective } \\
\text { cohort }\end{array}$ & EUA & $\begin{array}{l}\text { Pregnants } \\
>20 \text { weeks }\end{array}$ & 2127 & 9 & 31 & PCR & $4.7 \%$ & 6 \\
\hline $\begin{array}{l}\mathrm{Yu} \\
\text { et al. }{ }^{36}\end{array}$ & $\begin{array}{c}\text { Prospective } \\
\text { cohort }\end{array}$ & China & Pregnants & 300 & 0 & 10 & PCR & $11 \%(33 / 300)$ & 7 \\
\hline
\end{tabular}

n, number of subjects; loss, loss to follow-up; P, prevalence.

* loss of approximately $10 \%$.

\section{DISCUSSION}

CT genital infection is considered a potential risk factor for perinatal complications and has been the subject of numerous studies in recent decades. In this review, risk of PTL was found to be associated with CT infection, with a measure of RR of 1.51 [1.27, 1.80]. A more conservative assessment including only articles considered statistically homogeneous lowered the risk, $\mathrm{RR}=1.35[1.11,1.63]$, even though this assessment showed the highest risk of PTL delivery for pregnant women infected with CT. Blas et al. study ${ }^{13}$ was significant to this review due to sample size and confirmed this association as also seen in smaller studies. ${ }^{20,28,30,32,35}$ Studies suggest that treatment of CT in- fection reduces the morbidity of pregnancy. However data from Andrews et al..$^{20}$ do not confirm this fact, suggesting that gestational age at treatment and re-infection may also influence perinatal morbidity. ${ }^{37}$

Similarly, PROM was also more common in pregnant women infected with CT, OR $=1.13[0.95,1.34]$. However since the confidence interval includes the null value, the fact that CT infection increases the risk of PROM could not be confirmed. Kovacs et al. study, ${ }^{33}$ which was the most significant among the included, showed no significant difference in the association between CT infection and PROM. ${ }^{13,28,33,36}$ There is evidence that timely treatment of CT during antenatal care can significantly decrease the risk of PROM. That 
Table 2. Summary estimates of selected studies for association between Chlamydia infection and perinatal morbidity, according to the outcomes

\begin{tabular}{|c|c|c|c|c|c|c|c|c|}
\hline \multicolumn{9}{|c|}{ 2.1 Preterm labor } \\
\hline \multirow[b]{2}{*}{ Study } & \multicolumn{2}{|c|}{ Experimental } & \multicolumn{3}{|c|}{ Control } & \multirow{2}{*}{$\begin{array}{c}\text { Risk ratio } \\
\text { M-H, fixed, 95\% CI }\end{array}$} & \multirow{2}{*}{\multicolumn{2}{|c|}{$\begin{array}{c}\text { Risk ratio } \\
\text { M-H, fixed, 95\% CI }\end{array}$}} \\
\hline & Events & Total & Events & Total & Weight & & & \\
\hline Andrews et al. ${ }^{20}$ & 24 & 233 & 191 & 2123 & $25.3 \%$ & $1.14(0.77,1.71)$ & \multirow{9}{*}{ च } & \multirow{9}{*}{$\begin{array}{l}\square \\
\square \\
\square\end{array}$} \\
\hline Blas et al. ${ }^{13}$ & 76 & 735 & 181 & 2972 & $48.2 \%$ & $1.70(1.32,2.19)$ & & \\
\hline Gravett et al. ${ }^{28}$ & 17 & 47 & 64 & 535 & $0.0 \%$ & $3.02(1.94,4.71)$ & & \\
\hline Harrison et al. ${ }^{32}$ & 8 & 48 & 100 & 558 & $10.6 \%$ & $0.93(0.48,1.79)$ & & \\
\hline Jain et al. ${ }^{32}$ & 2 & 21 & 0 & 39 & $0.0 \%$ & $9.09(0.46,181.04)$ & & \\
\hline Martin et al. ${ }^{30}$ & 5 & 18 & 15 & 250 & $0.0 \%$ & $4.63(1.90,11.30)$ & & \\
\hline Silveira et al. ${ }^{35}$ & 11 & 97 & 254 & 1988 & $15.9 \%$ & $0.89(0.50,1.57)$ & & \\
\hline Total $(95 \%$ CI) & 1113 & & 7641 & & $100.0 \%$ & $1.35(1.11,1.63)$ & & \\
\hline Total events & 119 & & 726 & & & & & \\
\hline \multicolumn{7}{|c|}{$\begin{array}{l}\text { Heterogeneity: } \mathrm{Ch}^{2}=7.07, \mathrm{df}=3(\mathrm{p}=0.07) ; \mathrm{I}^{2}=58 \% \\
\text { Test for overall effect: } \mathrm{Z}=3.06(\mathrm{p}=0.002)\end{array}$} & $\begin{array}{lll}0.1 & 0.2 & 0.5 \\
\text { Experimental }\end{array}$ & $\begin{array}{lcc}2 & 5 & 10 \\
\text { Control }\end{array}$ \\
\hline
\end{tabular}

\subsection{Premature rupture of membrans}

\begin{tabular}{|c|c|c|c|c|c|c|c|c|}
\hline \multirow{3}{*}{$\begin{array}{l}\text { Study } \\
\text { Blas et al. }{ }^{13}\end{array}$} & \multicolumn{2}{|c|}{ Experimental } & \multicolumn{3}{|c|}{ Control } & \multirow{2}{*}{$\begin{array}{c}\text { Risk ratio } \\
\text { M-H, fixed, 95\% CI }\end{array}$} & \multirow{2}{*}{\multicolumn{2}{|c|}{$\begin{array}{c}\text { Risk ratio } \\
\text { M-H, fixed, 95\% CI }\end{array}$}} \\
\hline & \multirow{2}{*}{$\begin{array}{c}\text { Events } \\
49\end{array}$} & \multirow{2}{*}{$\begin{array}{c}\text { Total } \\
839\end{array}$} & \multirow{2}{*}{$\begin{array}{c}\text { Events } \\
130\end{array}$} & \multirow{2}{*}{$\begin{array}{l}\text { Total } \\
3378 \\
\end{array}$} & \multirow{2}{*}{$\begin{array}{c}\text { Weight } \\
27.1 \% \\
\end{array}$} & & & \\
\hline & & & & & & $1.50(1.09,2.06)$ & \multirow{7}{*}{ - } & \multirow{5}{*}{ - } \\
\hline Gravett et al. ${ }^{28}$ & 11 & 47 & 54 & 487 & $0.0 \%$ & $2.11(1.94,3.75)$ & & \\
\hline Harrison et al. ${ }^{32}$ & 4 & 39 & 50 & 447 & $4.2 \%$ & $0.92(0.35,2.41)$ & & \\
\hline Kovacs et al. ${ }^{33}$ & 70 & 352 & 1162 & 5809 & $68.8 \%$ & $0.99(0.80,1.23)$ & & \\
\hline Yu et al. ${ }^{36}$ & 10 & 33 & 33 & 267 & $0.0 \%$ & $2.45(1.33,4.50)$ & & \\
\hline Total $(95 \%$ CI) & \multicolumn{2}{|l|}{1240} & \multicolumn{2}{|l|}{9634} & $100.0 \%$ & $1.13(0.95,1.34)$ & & \multirow{2}{*}{ - } \\
\hline Total events & \multicolumn{2}{|l|}{123} & \multicolumn{3}{|l|}{1342} & & & \\
\hline \multicolumn{7}{|c|}{$\begin{array}{l}\text { Heterogeneity: } \mathrm{Ch}^{2}=7.07 ; \mathrm{df}=3(\mathrm{p}=0.07) ; \mathrm{I}^{2}=56 \% \\
\text { Test for overall effect: } \mathrm{Z}=1.34(\mathrm{p}=0.18)\end{array}$} & $\begin{array}{cc}0.01 & 0.1 \\
\text { Experimental }\end{array}$ & $\begin{array}{l}10100 \\
\text { Control }\end{array}$ \\
\hline
\end{tabular}

\subsection{Low birth weigh}

\begin{tabular}{|c|c|c|c|c|c|c|c|c|}
\hline \multirow{3}{*}{$\begin{array}{l}\text { Study } \\
\text { Berman et al. }^{31}\end{array}$} & \multicolumn{2}{|c|}{ Experimental } & \multicolumn{3}{|c|}{ Control } & \multirow{3}{*}{$\begin{array}{c}\text { Risk ratio } \\
\text { M-H, fixed, 95\% CI }\end{array}$} & \multirow{2}{*}{\multicolumn{2}{|c|}{$\begin{array}{c}\text { Risk ratio } \\
\text { M-H, fixed, 95\% CI }\end{array}$}} \\
\hline & \multirow{2}{*}{$\begin{array}{c}\text { Events } \\
11\end{array}$} & \multirow{2}{*}{$\begin{array}{c}\text { Total } \\
149 \\
\end{array}$} & \multirow{2}{*}{$\begin{array}{c}\text { Events } \\
37\end{array}$} & \multirow{2}{*}{$\begin{array}{l}\text { Total } \\
2123\end{array}$} & \multirow{2}{*}{$\begin{array}{c}\text { Weight } \\
25.3 \%\end{array}$} & & & \\
\hline & & & & & & & & - \\
\hline Blas et al. ${ }^{13}$ & 40 & 848 & 114 & 2972 & $48.2 \%$ & $1.41(0.99,2.00)$ & & 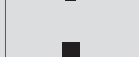 \\
\hline Gravett et al. ${ }^{28}$ & 15 & 47 & 73 & 535 & $0.0 \%$ & $2.12(1.33,3.38)$ & & \\
\hline Harrison et al..$^{32}$ & 4 & 39 & 51 & 558 & $10.6 \%$ & $1.03(0.39,2.79)$ & & 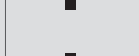 \\
\hline Jain et al. ${ }^{29}$ & 6 & 21 & 1 & 38 & $0.6 \%$ & $10.86(1.40,84.24)$ & & I \\
\hline Kovacs et al. ${ }^{33}$ & 24 & 359 & 303 & 5802 & $30.0 \%$ & $1.28(0.86,1.91)$ & & • \\
\hline Martin et al..$^{30}$ & 5 & 18 & 19 & 238 & $2.3 \%$ & $3.48(1.47,8.23)$ & & - \\
\hline Total $(95 \%$ CI) & 1481 & & 11141 & & $100.0 \%$ & $1.52(1.24,1.87)$ & & - \\
\hline Total events & 105 & & 598 & & & & & $\bullet$ \\
\hline $\begin{array}{l}\text { Heterogeneity: C } \\
\text { Test for overall }\end{array}$ & $\begin{array}{l}h^{2}=10.6 \\
\text { ffect: } Z=\end{array}$ & $\begin{array}{l}\mathrm{df}=6 \\
.06(\mathrm{p}\end{array}$ & $\begin{array}{l}\text { 0.10); } \mathrm{I}^{2} \\
0001)\end{array}$ & $44 \% \mathrm{z}$ & & & $\begin{array}{cc}0.01 & 0.1 \\
\text { Experimental }\end{array}$ & $\begin{array}{ll}10 & 100 \\
\text { Control }\end{array}$ \\
\hline
\end{tabular}


Table 2. Summary estimates of selected studies for association between Chlamydia infection and perinatal morbidity, according to the outcomes (Cont.)

\begin{tabular}{|c|c|c|c|c|c|c|c|c|}
\hline \multicolumn{9}{|c|}{ 2.4 Perinatal mortality } \\
\hline \multirow{3}{*}{$\begin{array}{l}\text { Study } \\
\text { Blas et al. }{ }^{13}\end{array}$} & \multicolumn{2}{|c|}{ Experimental } & \multicolumn{3}{|c|}{ Control } & \multirow{2}{*}{$\begin{array}{c}\text { Risk ratio } \\
\text { M-H, fixed, 95\% CI }\end{array}$} & \multirow{2}{*}{\multicolumn{2}{|c|}{$\begin{array}{c}\text { Risk ratio } \\
\text { M-H, fixed, 95\% CI }\end{array}$}} \\
\hline & \multirow{2}{*}{$\begin{array}{c}\text { Events } \\
7\end{array}$} & \multirow{2}{*}{\begin{tabular}{c|} 
Total \\
851 \\
\end{tabular}} & \multirow{2}{*}{$\begin{array}{c}\text { Events } \\
15 \\
\end{array}$} & \multirow{2}{*}{$\begin{array}{l}\text { Total } \\
3304\end{array}$} & \multirow{2}{*}{$\begin{array}{c}\text { Weight } \\
28.0 \% \\
\end{array}$} & & & \\
\hline & & & & & & $1.87(0.76,4.56)$ & & \\
\hline Harrison et al. ${ }^{32}$ & 0 & 37 & 3 & 430 & $2.6 \%$ & $1.62(0.09,30.79)$ & & - \\
\hline Jain et al. ${ }^{29}$ & 3 & 21 & 0 & 39 & $1.7 \%$ & $12.73(0.69,235.31)$ & & • \\
\hline Kovacs et al. ${ }^{33}$ & 13 & 349 & 118 & 5799 & $62.6 \%$ & $1.83(1.04,3.21)$ & & 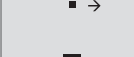 \\
\hline Martin et al. ${ }^{30}$ & 6 & 18 & 8 & 246 & $5.1 \%$ & $10.25(3.99,26.35)$ & & $\mathbf{a}$ \\
\hline Total (95\% CI) & 1276 & & 9918 & & $100.0 \%$ & $2.44(1.63,3.68)$ & & $\cdot$ \\
\hline Total events & 29 & & 144 & & & & & $\bullet$ \\
\hline $\begin{array}{l}\text { Heterogeneity: } \\
\text { Test for overall }\end{array}$ & $\begin{array}{l}\mathrm{A}^{2}=11.5 \\
\text { ffect: } Z=\end{array}$ & $\begin{array}{l}\mathrm{df}=4 \\
4.29(\mathrm{p}\end{array}$ & $\begin{array}{l}=0.02) ; \mathrm{I}^{2} \\
0001)\end{array}$ & $=65 \%$ & & & $\begin{array}{c}0.0101 \\
\text { Experimental }\end{array}$ & $\begin{array}{lr}10 & 100 \\
\text { Control }\end{array}$ \\
\hline
\end{tabular}

\subsection{Endometritis}

\begin{tabular}{|c|c|c|c|c|c|c|c|c|}
\hline \multirow[b]{2}{*}{ Study } & \multicolumn{2}{|c|}{ Experimental } & \multicolumn{3}{|c|}{ Control } & \multirow{2}{*}{$\begin{array}{c}\text { Risk ratio } \\
\text { M-H, fixed, 95\% CI }\end{array}$} & \multirow{2}{*}{\multicolumn{2}{|c|}{$\begin{array}{c}\text { Risk ratio } \\
\text { M-H, fixed, 95\% CI }\end{array}$}} \\
\hline & Events & Total & Events & Total & Weight & & & \\
\hline Berman et al. ${ }^{31}$ & 11 & 229 & 30 & 800 & $76.9 \%$ & $1.28(0.65,2.52)$ & \multirow{4}{*}{ - } & \multirow{4}{*}{ - } \\
\hline Harrison et al. ${ }^{32}$ & 2 & 76 & 25 & 869 & $23.1 \%$ & $0.91(0.22,3.79)$ & & \\
\hline Total (95\% CI) & 305 & & 1669 & & $100.0 \%$ & $1.20(0.65,2.20)$ & & \\
\hline Total events & 13 & & 55 & & & & & \\
\hline \multicolumn{7}{|c|}{$\begin{array}{l}\text { Heterogeneity: } \mathrm{Ch}^{2}=0.18 ; \mathrm{df}=1(\mathrm{p}=0.67) ; \mathrm{I}^{2}=0 \% \\
\text { Test for overall effect: } \mathrm{Z}=0.58(\mathrm{p}=0.56)\end{array}$} & $\begin{array}{c}0.010 .1 \\
\text { Experimental }\end{array}$ & $\begin{array}{l}10100 \\
\text { Control }\end{array}$ \\
\hline
\end{tabular}

\subsection{Abortion}

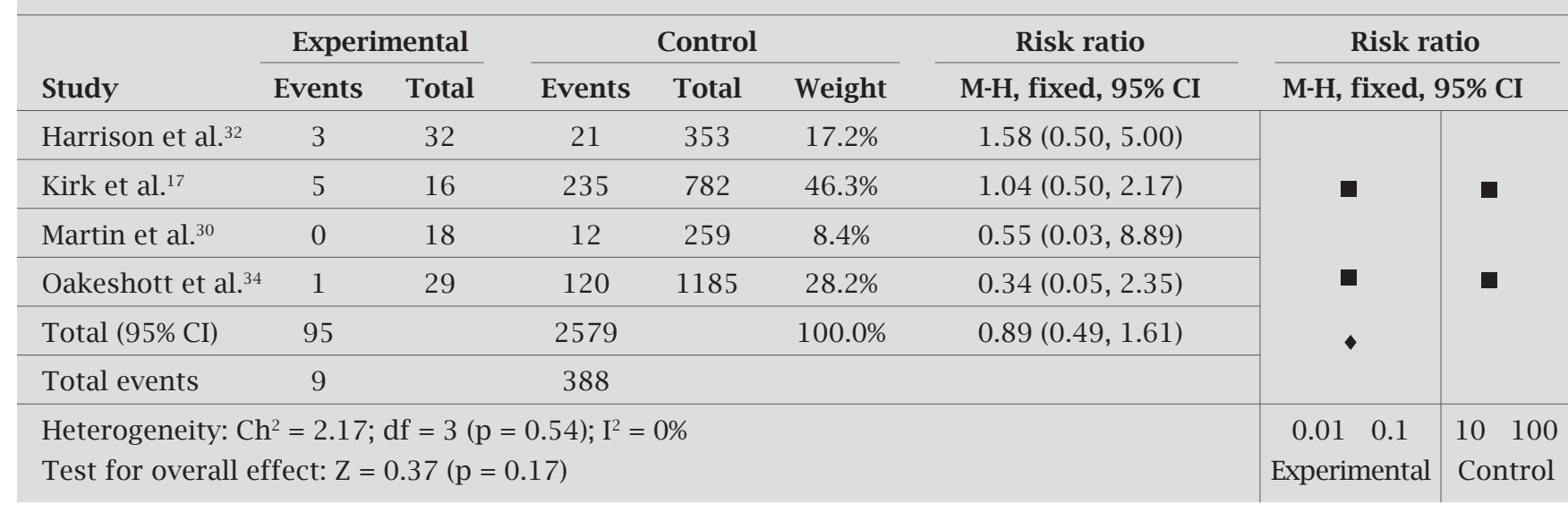


2695 articles identified in electronic databases and manual search

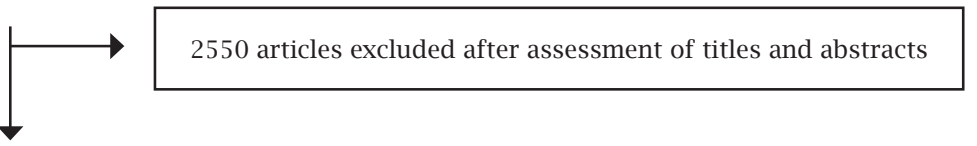

145 articles with full text assessment

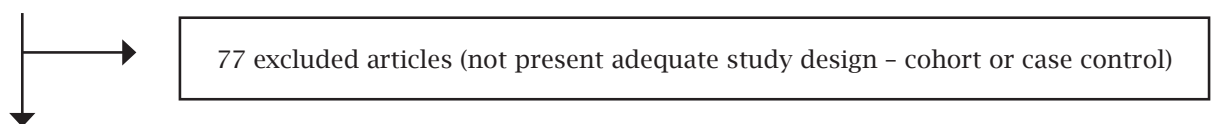

68 articles with full text assessment

56 excluded articles (not meet the selection criteria)

12 articles

Figure 1: Process used in the selection of articles from the search to the quality assessment.

being said, the effectiveness of treatment during pregnancy is $97 \%$ (92.9-99.2) using azithromycin, $95 \%$ (76.2-99.9) with amoxicillin and 64\% (44.1-81.4) with erythromycin. Such antibiotics are considered safe for use during the prenatal period. ${ }^{38}$

Pregnant women with CT infection also increased the risk of low birth weight, $\mathrm{RR}=1.52[1.24,1.87]$. All items involved in this review demonstrated an increased risk of CT infection during pregnancy, a fact statistically reinforced after the effect of the summary measure. Despite the fact that low birth weight may be brought about by several other causes, this data suggest that early diagnosis and treatment of CT infection during pregnancy can minimize the occurrence. ${ }^{13,29,30,32,33}$ However, even with appropriate treatment, $10-20 \%$ of infections persist or recur during the prenatal period, contributing to the emergence of adverse events in pregnancy even with appropriate treatment. Thus, follow-up tests may be needed to monitor infection. ${ }^{37}$

The rate of perinatal mortality, defined as a death from 20 weeks gestation until 28 days after birth, was significantly higher in pregnant women with CT infection, with a risk of $1.84[1.15,2.94]$. All articles showed increased risk, which supported the statistical significance after the summary measure and analysis of heterogeneity. ${ }^{13,29,30,32,33}$
With regard to abortion and endometritis associated with CT, summary measure did not show significant increased risk. Few papers have studied these factors and have shown opposing results. CT infection is highly prevalent among pregnant women. It can be assumed that studies with high methodological quality associated CT infection with an increased risk of prematurity, low birth weight and perinatal mortality. The publication of new studies could possibly strengthen the link being suggested in current studies. The significance of screening for this infection in pregnant women should be considered, especially when dealing with younger populations and risk factors for STDs. Thus, randomized clinical trials in treatment of CT infection in pregnant women are needed to evaluate the possibility of reducing this risk.

\section{ACKNOWLEDGEMENTS}

This study was financed by CNPQ - Edital MCT/CNPq $\mathrm{n}^{\circ}$ 70/2008 - Mestrado e Doutorado (\# 554784/2009-2).

\section{REFERENCES}

1. Al-Fouzan A, Al-Mutairi N. Overview of incidence of sexually transmitted diseases in Kuwait. Clin Dermatol. 2004;22(6):509-512. 
2. Centers for Disease, Control and Prevention. Chlamydia screening among sexually active young female enrollees of health plans - United States, 1999-2001. MMWR Morb Mortal Wkly Rep. 2004;53(42):983-985.

3. Chotnopparatpattara P, Limpongsanurak S, Wongprechasawas A. The prevalence of Chlamydia trachomatis infection in pregnant Thai women. J Med Assoc Thai. 2003;86 Suppl 2:S399-403.

4. Currie MJ, Bowden FJ. The importance of chlamydial infections in obstetrics and gynaecology: an update. Aust $\mathrm{N} Z \mathrm{~J} \mathrm{Ob-}$ stet Gynaecol. 2007;47(1):2-8.

5. Centers for Disease Control and Prevention. Sexually Transmitted Disease Surveillance, 2005. 2006.

6. World Health Organization. Global prevalence and incidence of selected curable sexually transmitted diseases: overview and estimates.Geneva; 2001.

7. Beagley KW, Timms P. Chlamydia trachomatis infection: incidence, health costs and prospects for vaccine development. J Reprod Immunol. 2000;48(1):47-68.

8. Manavi K. A review on infection with Chlamydia trachomatis. Best Pract Res Clin Obstet Gynaecol. 2006;20(6):941-951.

9. Barcelos MR, Vargas PR, Baroni C, Miranda AE. Genital infections in women attending a Primary Unit of Health: prevalence and risk behaviors. Rev Bras Ginecol Obstet. 2008;30(7):349-354.

10. Jalil EM, Pinto VM, Benzaken AS, et al. Prevalência da infecção por clamídia e gonococo em gestantes de seis cidades brasileiras. Rev Bras Ginecol Obstet. 2008;30:614-619.

11. Bebear C, de Barbeyrac B. Genital Chlamydia trachomatis infections. Clin Microbiol Infect. 2009;15(1):4-10.

12. Avasthi K, Garg T, Gupta S, Grewal RK, Ram S. A study of prevalence of Chlamydia trachomatis infection in women with first trimester pregnancy losses. Indian J Pathol Microbiol. 2003;46(1):133-136.

13. Blas MM, Canchihuaman FA, Alva IE, Hawes SE. Pregnancy outcomes in women infected with Chlamydia trachomatis: a population-based cohort study in Washington State. Sex Transm Infect. 2007;83(4):314-318.

14. Peipert JF. Clinical practice. Genital chlamydial infections. N Engl J Med. 2003;349(25):2424-2430.

15. Bakken IJ, Skjeldestad FE, Nordbo SA. Chlamydia trachomatis infections increase the risk for ectopic pregnancy: a population-based, nested case-control study. Sex Transm Dis. 2007;34(3):166-169.

16. Bebear C, de Barbeyrac B. Genital Chlamydia trachomatis infections. Clin Microbiol Infect. 2009;15(1):4-10.

17. Kirk E, Bora S, Van Calster B, et al. Chlamydia trachomatis infection in patients attending an Early Pregnancy Unit: prevalence, symptoms, pregnancy location and viability. Acta Obstet Gynecol Scand. 2008;87(6):601-607.

18. Fitz Simmons J, Callahan C, Shanahan B, Jungkind D. Chlamydial infections in pregnancy. J Reprod Med. 1986;31(1):19-22.

19. Much DH, Yeh SY. Prevalence of Chlamydia trachomatis infection in pregnant patients. Public Health Rep. 1991;106(5):490-493.

20. Andrews WW, Klebanoff MA, Thom EA, et al. Midpregnancy genitourinary tract infection with Chlamydia trachomatis: association with subsequent preterm delivery in women with bacterial vaginosis and Trichomonas vaginalis. Am J Obstet Gynecol. 2006;194(2):493-500.

21. Chamani-Tabriz L, Tehrani MJ, Akhondi MM, et al. Chlamydia trachomatis prevalence in Iranian women attending obstetrics and gynaecology clinics. Pak J Biol Sci. 2007;15;10(24):44904494 .
22. Cheney K, Wray L. Chlamydia and associated factors in an under 20s antenatal population.Aust N Z J Obstet Gynaecol. $2008 ; 48(1): 40-43$.

23. Leite R. Infecção cervical causada por Chlamydia Trachomatis em gestantes. Estudo de prevalência e fatores de risco. Rev Bras Gin Obst. 2001;23:58-58.

24. Baczynska A, Hvid M, Lamy P, Birkelund S, Christiansen G, Fedder J. Prevalence of Mycoplasma genitalium, Mycoplasma hominis and Chlamydia trachomatis among Danish patients requesting abortion. Syst Biol Reprod Med. 2008;54(3):127-134.

25. Mardh PA. Influence of infection with Chlamydia trachomatis on pregnancy outcome, infant health and life-long sequelae in infected offspring. Best Pract Res Clin Obstet Gynaecol. 2002;16(6):847-864.

26. Kadzhaia D, Merabishvili N. Prevalence and risk factors for Chlamydia trachomatis infection in pregnant women. Georgian Med News. 2005(129):33-36.

27. Vaz FAC, Ceccon MEJ, Diniz EMA. Infecção por Chlamydia trachomatis no período neonatal: aspectos clínicos e laboratoriais. Experiência de uma década: 1987-1998. Rev Ass Med Bras. 1999;45:303-311.

28. Gravett MG, Nelson HP, DeRouen T, Critchlow C, Eschenbach DA, Holmes KK. Independent associations of bacterial vaginosis and Chlamydia trachomatis infection with adverse pregnancy outcome. JAMA. 1986;256(14):1899-1903.

29. Jain A, Nag VL, Goel MM, Chandrawati, Chaturvedi UC. Adverse foetal outcome in specific IgM positive Chlamydia trachomatis infection in pregnancy. Indian J Med Res. 1991;94:420-423.

30. Martin DH, Koutsky L, Eschenbach DA, et al. Prematurity and perinatal mortality in pregnancies complicated by maternal Chlamydia trachomatis infections. JAMA. 1982;247(11):1585-1588.

31. Berman SM, Harrison HR, Boyce WT, Haffner WJ, Lewis M, Arthur JB. Low birth weight, prematurity, and postpartum endometritis. Association withprenatal cervical $M y$ coplasma hominis and Chlamydia trachomatis infections. JAMA. 1987;257(9):1189-94.

32. Harrison HR, Alexander ER, Weinstein L, Lewis M, Nash M, Sim DA. Cervical Chlamydia trachomatis and mycoplasmal infections in pregnancy. Epidemiology and outcomes. JAMA. 1983;250(13):1721-1727.

33. Kovacs L, Nagy E, Berbik I, Meszaros G, Deak J, Nyari T. The frequency and the role of Chlamydia trachomatis infection in premature labor. Int J Gynaecol Obstet. 1998;62(1):47-54.

34. Oakeshott P, Hay P, Hay S, Steinke F, Rink E, Kerry S. Association between bacterial vaginosis or chlamydial infection and miscarriage before 16 weeks' gestation: prospective community based cohort study. BMJ. 2002;325(7376):1334.

35. Silveira MF, Ghanem KG, Erbelding EJ, et al. Chlamydia trachomatis infection during pregnancy and the risk of preterm birth: a case-control study. Int J STD AIDS. 2009;20(7):465-469.

36. Yu J, Wu S, Li F, Hu L. Vertical transmission of Chlamydia trachomatis in Chongqing China. Curr Microbiol. 2009;58(4):315-320.

37. Miller JM, Maupin RT, Nsuami M. Initial and repeat testing for Chlamydia during pregnancy. Am Fam Physician. 2005;18:231-235.

38. Rahangdale L, Guerry S, Bauer HM, et al. An observational cohort study of Chlamydia trachomatis treatment in pregnancy. Sex Transm Dis. 2006;33(2):106-110. 